\section{Emilio Zola y la influencia de la Medicina en su obra La Fortuna de los Rougon-Macquart}

\author{
MARCELO MIRANDA C. ${ }^{1}$, MARÍA FLORENCIA ALAMOS ${ }^{2,3, a}$
}

\section{The influence of medicine in Emile Zola's "Fortune of the Rougon-Macquart"}

Emile Zola is one of the greatest writers in universal literature. In his important series of novels called "The Fortune of the Rougon-Macquart", Zola shows a surprising medical knowledge even though he did not have a formal medical education. We highlight not only his outstanding literary talent, but also the scientific relevance of the tremendous contribution to the medical field that can be extracted from his work. In this series, which describe the history of five generations within a large family suffering from neuropsychiatric and general pathologies, Zola emphasizes the hereditary component of several diseases. These observations probably place him as the first novelist who made an explicit emphasis on the power of inheritance in human behavior. He also mentions for the first time several medical aspects that were seldom addressed in the scientific literature of the time, demonstrating the genius of the writer, his outstanding power of observation and the rigorous preparation with which he wrote his work.

(Rev Med Chile 2019; 147: 1329-1334)

Key words: Genetics; History of Medicine; Medicine in Literature.

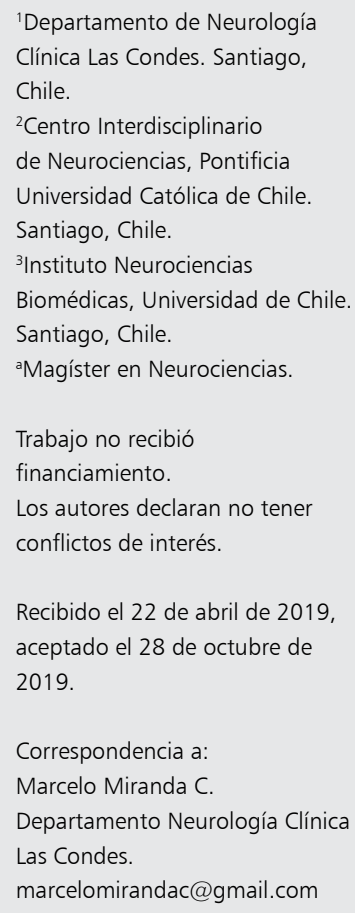

\section{"El novelista está formado por un observador y un experimentador"}

\author{
Emile Zola
}

E milio Zola (París, 1840-París, 1902) (Figura 1) permanece en la historia como uno de los mejores escritores franceses y de los más grandes autores de la literatura universal. Zola es el fundador del estilo naturalista caracterizado por su crudeza en mostrar la realidad con el fin de motivar a mejorarla, y ha dejado entrever de forma extraordinaria (comparable a Dostoievski) la pobreza, miseria y degradación humana, ambición y el poder del dinero. El mismo Zola decía que lo que él hacía era abrir la ventana de su casa y describir lo que veía.
Su compromiso por la justicia en el célebre caso Dreyfus le llevó al exilio y fue causa directa de su muerte precoz ${ }^{1-3}$.

En este artículo se hace énfasis en un aspecto casi desconocido de Zola, su asombroso conocimiento médico, sin tener ninguna educación formal en esta ciencia". En su obra maestra " $L a$ Fortuna de los Rougon-Macquart ", una extensa serie de 20 novelas publicadas desde 1871 hasta $1893^{7-12}$ que le reportó fama y dinero, Zola describió la distinta suerte de los miembros de una familia de provincia, en la cual algunos logran llegar a París alcanzando una muy distinta fortuna. En esta obra, Zola describió claramente diversas patologías heredadas o no por los miembros de cinco generaciones: menciona casos de demencia, psicopatías, epilepsia, alcoholismo, delirium tremens, tics, ataxias, cardiopatía coronaria, etc. 




Figura 1. Emile Zola (París 1840) se aficionó a la fotografía en 1888 y llegó a realizar cerca de 7.000 fotos hasta 1902, año de su muerte. En esta foto él mismo se fotografía (una de las primeras "selfies") (Copyright: www.bridgemanimages.com). Compró una docena de cámaras y montó tres laboratorios para revelar. En la foto, el escritor con su Box 7.

El protagonista de la novela final de la serie (" $E l$ Doctor Pascal") es un médico que practica incluso el uso de placebo y que utiliza lo que podría considerarse un precursor de terapia restauradora celular $^{12}$.

\section{Datos biográficos}

Emilio Zola fue hijo de Émilie Aubert y de el ingeniero italiano Francesco Zola ${ }^{5}$. Creció en Aix-en-Provence, ciudad conocida como Plassans en sus obras ${ }^{1,3,5}$. Durante el período escolar fue compañero del pintor Paul Cézanne, con quien forjó una gran amistad, y recibió precozmente influencias de la literatura romántica ${ }^{5}$. Cuando Zola tenía 7 años su padre fallece. Un año más tarde se traslada junto a su madre a vivir a París. Estudió leyes, sin embargo, luego de fracasar en su examen de graduación y de enfrentarse a una delicada situación económica decidió abandonar sus estudios. Primero trabajó como empleado de aduanas y en luego en el área de publicidad de la editorial Hachette, de la cual fue despedido en $1866^{1,2,5}$.

Se rodeó de importantes escritores naturalistas de la época, entre los que destacaron Maupassant y los hermanos Goncourt. Fue también gran amigo de los pintores impresionistas Cézanne, Manet, Monet, Renoir y Pissarro ${ }^{1,2,3 \text { y } 5}$.

El libro Introducción a la medicina experimental, de Claude Bernard, le sirvió de inspiración y ejemplo para el relato riguroso de una serie de novelas que tratan de la historia natural de una familia durante 5 generaciones a lo largo del Segundo Imperio.

Así nació la monumental serie "Los Rougon-Macquart", integrada por La fortuna de los Rougon (1871), La jauría (1871), El vientre de París (1873), La conquista de Plassans (1874), La caída del Abate Mouret (1875), Su excelencia Eugène Rougon (1876), La taberna (1877), Una página de amor (1878), Nana (1879) (Figura 2), Lo que se

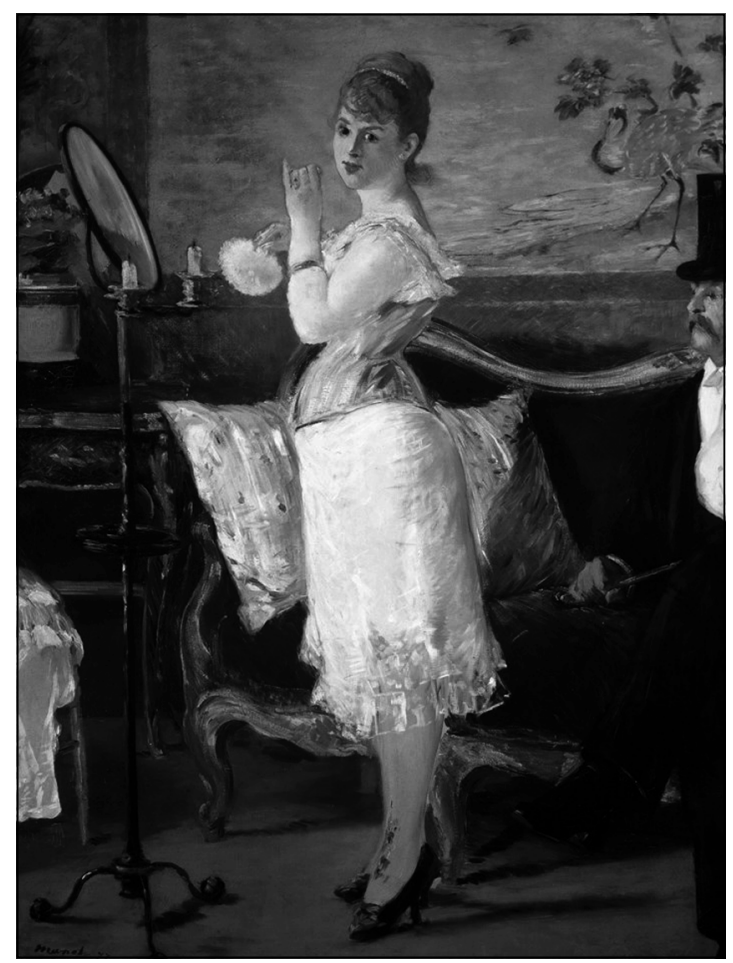

Figura 2. Nana por el pintor Édouard Manet (Kunsthalle, Hamburg, Alemania). 
gasta (1882), El paraíso de las damas (1883), La alegría de vivir (1884), Germinal (1885), La obra (1886), La tierra (1887), El sueño (1888), La bestia humana (1890), El dinero (1891), La derrota (1892) y El Doctor Pascal (1893). En las 20 novelas que comprende la serie, Zola trazó la genealogía de más de doscientos personajes y sus textos recibieron comentarios muy positivos, así como también fuertes críticas ${ }^{5}$.

\section{La Serie de los Rougon-Macquart}

"Los Rougon-Macquart" es un conjunto de 20 novelas escrito por Zola que imprimen el éxito del movimiento literario naturalista ${ }^{1,2,6}$. Esta obra esta inspirada en la famosa serie "La Comedia Humana" del autor francés Honoré de Balzac ${ }^{6}$. En "Los Rougon-Macquart" Zola intenta estudiar los defectos hereditarios de una familia a lo largo de cinco generaciones (Figura 3), comenzando con
Adelaida Fouque, nacida en 1768, y terminando en 1893 con el hijo que tuvieron el Dr. Pascal Rougon y su sobrina Clotilde 6 . Esto se expresa de forma inmediata en la "La fortuna de los Rougon" (la primera novela de la serie): "Quiero explicar cómo una familia, un pequeño grupo de seres humanos, se comporta en una sociedad, desarrollándose para engendrar diez o veinte individuos que parecen, a primera vista, profundamente distintos, pero que el análisis muestra intimamente ligados los unos a los otros. La herencia tiene sus leyes, como la gravedad"5. En esta serie de obras quiso también describir la sociedad durante el Segundo Imperio de manera exhaustiva, sin olvidar ninguno de sus componentes, dando cabida a las grandes transformaciones que se producen en esta época: urbanismo parisino (encargo de Napoleón III al barón Haussman para hacer los grandes bulevares $)^{9}$, grandes almacenes (en "El paraíso de las mujeres"), desarrollo del ferrocarril (en "La bestia humana"), aparición del sindicalismo moderno ${ }^{8}$, etc.

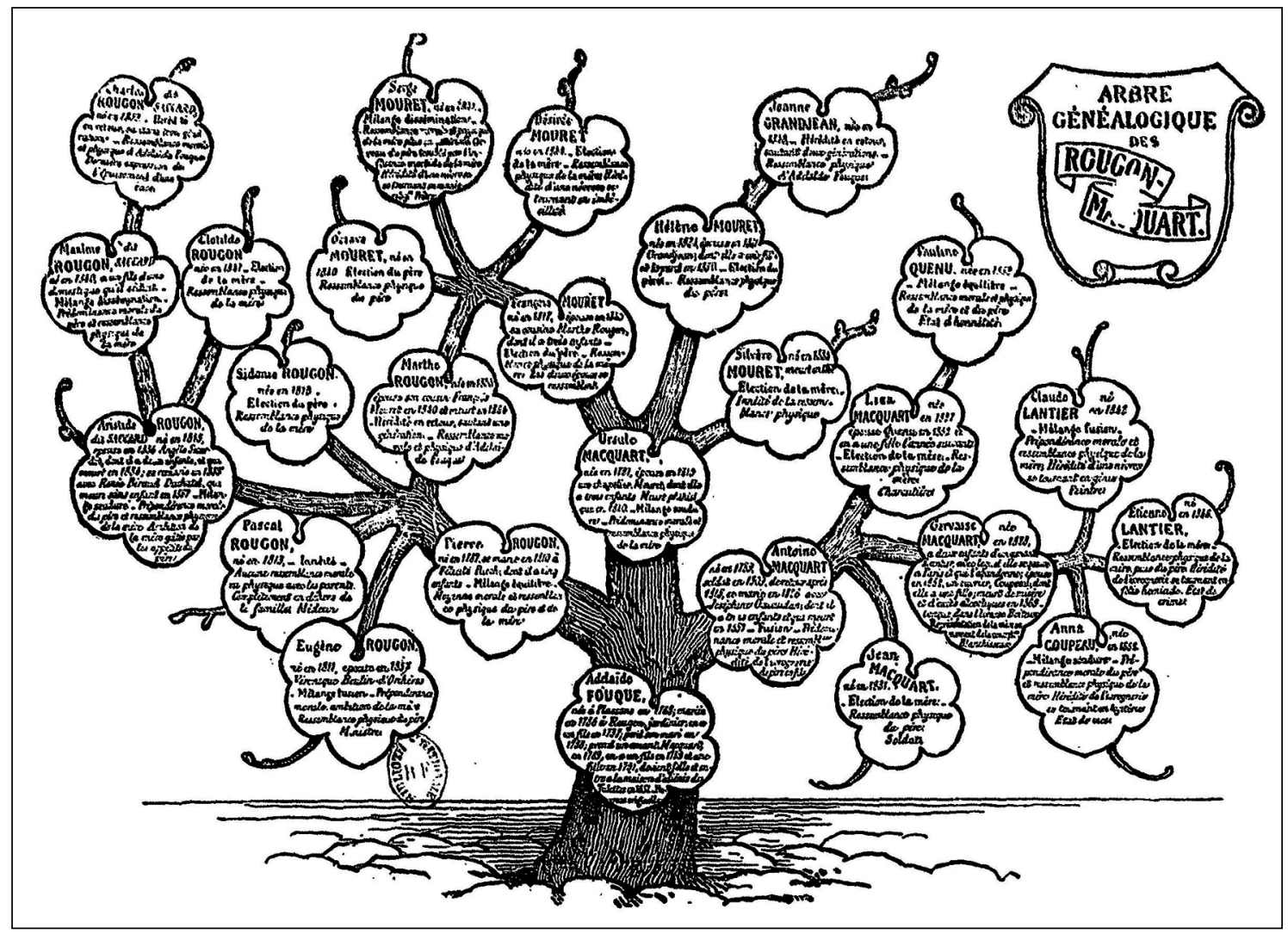

Figura 3. Árbol genealógico de la familia Rougon-Macquart diseñado por el propio Zola (Meisterdrucke. French Collection. Villach, Austria). 
Las obras más representativas y exitosas de esta serie son Germinal, La Taberna y $\mathrm{Nana}^{9-10}$. Si bien en varias de ellas Zola hizo despliegue de conocimientos médicos, es El Dr. Pascal, la última novela de la serie, la que sobresale por este aspecto ${ }^{12}$. Aquí, Zola se retrata en parte en la personalidad del médico de provincia, Dr. Pascal, quien es un atípico representante de la familia Rougon-Macquart, en el sentido de ser el más honesto y equilibrado de todos, salvo en su relación amorosa e incestuosa con su sobrina, quien incluso le da un hijo. El Dr. Pascal es un estudioso que lleva un registro genealógico actualizado con gran detalle de su extensa familia (Figura 3), anotando todos los rasgos físicos, patológicos, de personalidad y la forma en que se han heredado por cinco generaciones. Destaca la abuela, quien padece de demencia degenerativa, convulsiones, lenguaje muy limitado y está confinada en un sanatorio. También hay sobrinos alcohólicos, otros atáxicos y otro miembro de la familia que presenta un grave retraso mental. Para aliviar los males de sus pacientes, el Dr. Pascal realizaba experimentos en su casa-laboratorio con un producto que obtiene de un macerado de cerebro de cordero y que inyecta a sí mismo y a sus pacientes afectos de diversos cuadros como agotamiento o excesiva agresividad. Zola describió en estos términos cómo el Dr. Pascal llegó a provocar una embolia y la muerte a un paciente en el momento de inyectarlo: "había ido a dar una inyección a Lafouasse, el posadero, cuya ataxia había empeorado, la mala fortuna habia querido que la jeringa recogiera una partícula impura del filtrado. Para colmo de males el pinchazo había cogido una vena. Sintíose enseguida inquieto al ver palidecer al posadero, cubierto con sudor. Seguidamente, lo comprendió todo, al sobrevenir la muerte instantáneamente, fulminante dejando al infeliz con labios amoratados y el rostro negro. Se trataba sin duda de una embolia, que tan solo podía atribuir a la insuficiencia de sus preparaciones"12. Este tratamiento podría considerarse como una descripción pionera de lo que hoy conocemos como terapia celular, concepto introducido formalmente recién en 1930 por el médico sueco Paul Niehans ${ }^{13}$. Interesantemente, los resultados de Niehans tampoco fueron satisfactorios, reportándose efectos adversos que iban desde las reacciones alérgicas hasta la muerte ${ }^{13}$. Luego de este fracaso con el uso de esta terapia innovadora para la época, decidió solo usar placebo en forma de agua que inyecta a algunos pacientes, logrando en algunos de ellos y en sí mismo una mejoría transitoria. Zola, al usar el término embolia, hace muy probable que estuviera familiarizado con los trabajos del médico alemán Rudolf Virchow, quien hacía ya casi 50 años había introducido tal concepto y quien era ya muy conocido en Francia ${ }^{14}$.

El Dr. Pascal, protagonista de esta última novela de la serie, comenzó a sufrir de una cardiopatía coronaria expresada en típicos episodios de angor pectoris que Zola describió con gran detalle y que lleva al protagonista a morir de un infarto al miocardio.

Zola puso en palabras del Dr. Pascal su teoría que la extensa familia heredaba diversos rasgos patológicos ya sea mentales o físicos que se traspasaban de generación en generación, pero en lo cual también influía el medio ambiente. Zola fue fuertemente influenciado por sus lecturas de las obras de Charles Darwin, del fisiólogo Claude Bernard y de los primeros trabajos en genética publicados en la época ${ }^{1-3}$. Las obras de Gregorio Mendel, publicadas en 1866, no fueron reconocidas internacionalmente hasta 1900, lo que hace muy improbable que Zola las hubiera conocido. En esta última obra de su serie hizo mención en varias oportunidades de la palabra genética, dando a entender que existen "factores" que se traspasaban de padres a hijos y que estaban en las células germinativas. Pasarían 10 años antes que fuera acuñado el término gen y genética para señalar estos factores y la ciencia dedicada a ellos, por Wilhem Johannsen y William Bateson, respectivamente $^{15}$.

En "La Taberna", como su nombre sugiere, describió los estragos del alcohol en una pobre familia trabajadora en París y en el ambiente de la época ${ }^{10}$. Gervaise, quien es planchadora y muy empeñosa inicialmente, se rodea consecutivamente de dos hombres que solo se aprovechan de ella y se vuelven alcohólicos. Pese a ello, Gervaise vive con ellos y los mantiene. Uno de los hombres, Coupon, comenzó a desarrollar, debido al alcohol, severos temblores en las extremidades, llevándole a varios episodios de delirium tremens los que finalmente le conducen a la muerte. Zola describió en gran detalle estos episodios de agitación psicomotora, que presentaban alucinaciones visuales, hiperpirexia, intensos movimientos anormales y convulsiones. Coupon es atendido por un médico interno y otro médico de rango superior que toman nota 
de toda la evolución del delirium, pero no logran evitar el desenlace fatal ${ }^{8}$. Por otro lado, Gervaise, luego de tener éxito en su negocio de lavado y planchado de ropa, cae también en el alcoholismo y fallece en severa desnutrición. Gervaise y Coupon tuvieron como hija a Nana, protagonista de un volumen dedicado a ella. Nana, criada en ese tóxico ambiente, precozmente se hace prostituta y actriz teatral. Gracias a sus conquistas Nana logra un ascenso social. Sin embargo, su codicia desmedida por el dinero, inestabilidad afectiva y promiscuidad la conducen al ocaso precozmente muriendo de viruela ${ }^{7}$.

No tenemos fuentes para indicar con precisión cómo Zola obtuvo el conocimiento médico que despliega en su colosal serie. Si bien Zola conoció personalmente en alguna velada a Charcot, el padre de la Neurología, no acudió a sus "pasos prácticos" con pacientes, como lo hizo Guy de Maupassant ${ }^{16}$. Solo podemos inferir, entonces, que su conocimiento proviene de lecturas y una extraordinaria capacidad de observación, sumada a una pasión por el detalle y no dejar nada a la improvisación. Una clara muestra de esto es el contexto en que escribe su novela "La bestia humana". En ella, la gran acción ocurre en trenes en marcha, por lo que Zola se preparó exhaustivamente para escribirla haciendo múltiples viajes en trenes previamente, conociendo en detalle cómo funcionaban. En otra novela, "El Dinero", hizo un plano con gran detalle del edificio que representaba la Bolsa de Valores de París. En "Germinal”, ambientada en las minas de carbón del norte de Francia, cerca de Bélgica, acudió personalmente a las minas y desciende a las profundidades de las excavaciones para conocer mejor las difíciles condiciones de trabajo de los mineros 8 .

Vemos cómo Zola en sus propias palabras aplicaba el rigor científico de investigación directa en terreno (mercados, burdeles, minas, almacenes, etc.) con una cuidadosa observación y registro en notas para su posterior uso en sus obras.

Así Zola cumplía con el objetivo del naturalismo: hacer una denuncia de la injusticia -documentada con investigación en terreno y en el sitio del fenómeno bajo análisis-y presentar al público sus hallazgos en forma literaria con una prosa elegante, aplicando la ciencia y el positivismo al idealismo de anhelo para la justicia. Sin duda, Zola intentó aplicar el método científico a su prosa meticulosa en coloridas y detalladas descripciones de la sociedad y sus clases sociales ${ }^{1,2,4}$.

En el año 1898 Zola escribió el artículo-manifiesto " $; Y o$ acuso!", defendiendo la inocencia del capitán judío Alfred Dreyfus, acusado por militares antisemitas de alta traición a la patria ${ }^{5}$. Este artículo le significó el destierro a Inglaterra y su asesinato en 1902 en mano de fanáticos antisemitas: uno de ellos bloqueó la ventilación de una estufa de su dormitorio y Zola murió asfixiado con monóxido de carbono (Figura 4$)^{1-5}$. Tras la muerte de Zola y gracias a todo el proceso de exoneración que él inició, Dreyfus fue absuelto ${ }^{5}$.

Zola llevó una doble vida amorosa desde 1888: se enamoró de la costurera de su esposa llamada Jeanne Rozerot, 27 años menor y quien le dio dos hijos, los que no pudo tener con su pareja legal. Uno de ellos, Jacques, se tituló de médico y

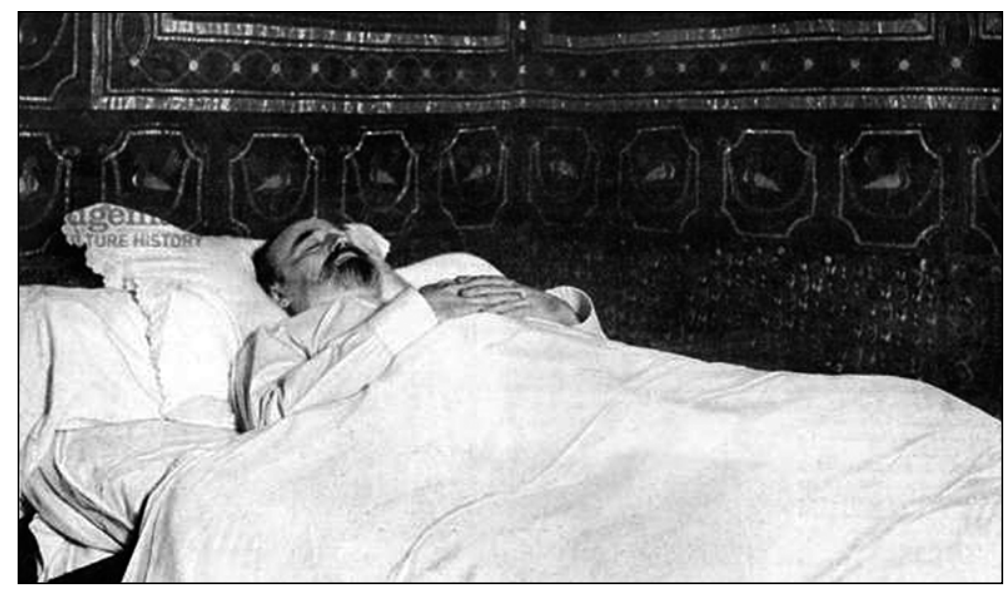

Figura 4. Zola en su lecho de muerte 1902 (imagen dominio público). 
ejerció la medicina. Su esposa finalmente aceptó la relación y logró que sus hijos ilegítimos llevaran el apellido Zola luego de la muerte del autor en 1902. Ambas parejas de Zola se reconciliaron y acudieron al traslado de los restos del autor en forma definitiva al Panteón de París, lugar de las máximas glorias francesas $s^{1,2,3}$.

En suma, Zola al igual que otros grandes escritores como Maupassant, Dickens, Proust ${ }^{16-18}$, etc, enriqueció sus trabajos con minuciosas descripciones médicas gracias a una sorprendente capacidad de observación y a una exhaustiva preparación. Probablemente, Zola sea el primer novelista que hizo un marcado énfasis en el poder de la herencia en la conducta humana. El aspecto médico en las obras concebidas así, sin duda que contribuyó a la magnificencia y trascendencia de este legado cultural.

\section{Referencias}

1. Schom A. Émile Zola: A Biography. San Francisco: Sharpe Books; 2018.

2. Hemmings FWJ. The Life and Times of Emile Zola. London: Bloomsbury Reader; 2011.

3. Zola D. Emilio Zola. Lo que cuenta su hija. Madrid: Editorial Zeus; 1931.

4. Koehler P. About Medicine and the Arts. Charcot and French Literature at the fin-de-siècle, J Hist Neurosci 2001; 10: 1, 27-40.

5. Émile Zola. (2019, mayo 29). EcuRed. Consultado el
20:03, agosto 26, 2019 en https://www.ecured.cu/index. php?title=\%C3\%89mile_Zola\&oldid=3387098.

6. Les Rougon-Macquart. (2019, 13 de agosto). Wikipedia, La enciclopedia libre. Fecha de consulta: 15:55, agosto 24, 2019 desde https://es.wikipedia.org/w/index.php?title=Les_Rougon-Macquart\&oldid=118225116

7. Zola E. La Fortuna de los Rougon. Barcelona: Editorial Alba; 2007.

8. Zola E. Germinal. Barcelona: Editorial Lorenzana; 1965.

9. Zola E. Nana. Barcelona: Editorial Edhasa; 1968.

10. Zola E. La taberna. Barcelona: Editorial Lorenzana; 1969.

11. Zola E. La Jauría. Barcelona: Editorial Alba; 2006.

12. Zola E. El Dr. Pascal. Barcelona: Editorial Lorenzana; 1965.

13. Abgrall J M. Healing or stealing? Medical Charlatans in the new age. New York: Algora publishing; 2001.

14. Mazana J.S. Rudolph Virchow in the centenary of his death. An Med Interna 2002; 19 (12): 649-50.

15. Knippers R. Eine Kurze Geschichte der Genetik. Springer. 2017.

16. Miranda M, Roa N, Bustamante ML. Guy de Maupassant: aspectos médicos de su creativa y desenfrenada vida. Rev Med Chile 2012; 140: 524-9.

17. Miranda M. Charles Dickens y su aporte a la medicina: en el bicentenario de su nacimiento. Rev Med Chile 2012; 140: 826-7.

18. Miranda, M. Marcel Proust: el rol de su enfermedad y la Medicina en la vida y obra del autor de "A la busca del tiempo perdido", a un siglo de su creación. Rev Med Chile 2009; 137: 433-7. 